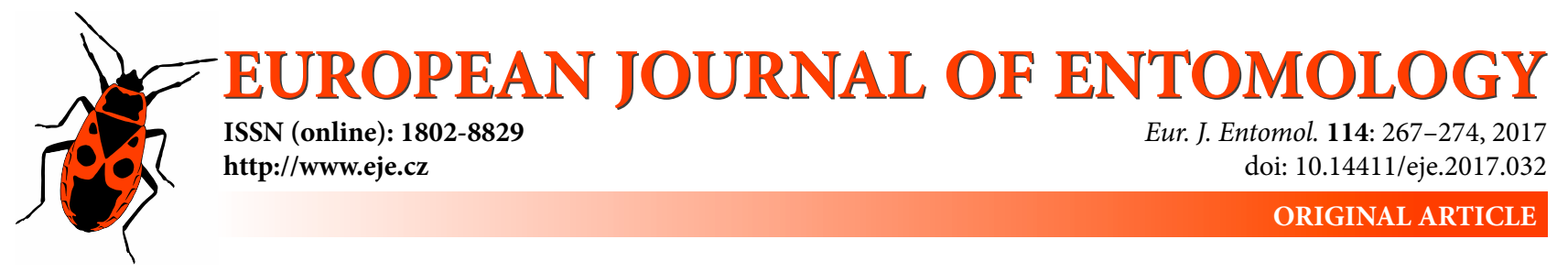

\title{
Mating advantage of short-winged over long-winged adult males in the cricket Velarifictorus ornatus (Orthoptera: Gryllidae)
}

\author{
Lv-QuAn ZHAO ${ }^{1}$, HuAl-Lin CHAI ${ }^{2}$, Hong-Jun WU² and DAO-Hong ZHU ${ }^{2, *}$ \\ ${ }^{1}$ Co-innovation Center for Sustainable Forestry in Southern China, College of Forestry, Nanjing Forestry University, Nanjing, \\ China; e-mail: zhaolvquan80@163.com \\ ${ }^{2}$ Laboratory of Insect Behavior and Evolutionary Ecology, Central South University of Forestry and Technology, Changsha, \\ China; e-mails: chaihuailin@163.com, wuling9802@aliyun.com, daohongzhu@yeah.net
}

Key words. Orthoptera, Gryllidae, Velarifictorus ornatus, mating competition, sing song, trade-off, wing polymorphism

\begin{abstract}
The trade-off between flight capability and reproduction is well known in adult males of insects with wing dimorphism but the reproductive advantage of short-winged (SW) males over long-winged (LW) males appears to vary across insect taxa. In the present study, we determined the difference in the mating ability of SW and LW males of Velarifictorus ornatus (Orthoptera: Gryllidae) in order to evaluate whether the SW male morph has a reproductive advantage. We found that the choice of a mate depended on the female. Compared with LW males, SW males had an obvious mating advantage when both SW and LW males courted females simultaneously, and that dealation significantly enhanced the mating ability of LW males. Losing the ability to produce songs reduced the mating advantage of SW males, thereby indicating that the greater mating advantage of SW males was related to the attractiveness of the song. In addition, the difference in the mating ability of LW and SW males was not related to body size or age. These results indicate that SW males of $V$. ornatus have a mating advantage over their LW counterparts because their underdeveloped flight muscles allow them to devote more resources to reproduction.
\end{abstract}

\section{INTRODUCTION}

In the phylum Arthropoda, the evolution of wings occurred uniquely in insects and it is probably one of the most important factors responsible for their success because it must have given them a great advantage over other organisms due to their increased capacity for foraging, searching for mates and new habitats, and escaping from predators (Tanaka, 1994). However, despite these advantages, some species have lost their capacity for flight by reducing the size of their wings or losing them completely during the course of evolution. In addition, some have become dimorphic for flight without any apparent modification of the wings, whereas others shed their wings after adult emergence (Tanaka, 1994). Wing dimorphism is a common feature of many species of insects, especially those belonging to the orders Orthoptera, Hemiptera, Homoptera and Blattaria (Harrison, 1980; Roff, 1986). A widely accepted hypothesis is that a trade-off exists between the capacity for flight and reproduction in insects with wing dimorphism (Roff \& Fairbairn, 1991). Substantial empirical evidence supports this hypothesis in females, where flightless females tend to have higher fecundity than those that are capable of flight (Roff, 1986; Zera \& Denno, 1997; Guerra, 2011)

Understanding the ecological significance of dispersal polymorphism requires that fitness differences be documented between the wing forms of both sexes (Langellotto et al., 2000). However, a small number of studies have failed to detect this trade-off in males, e.g., similar sized gonads (Zhao et al., 2010), similar mating competitiveness (Sack \& Stern, 2007) and similar length of adult life (Mishiro et al., 1994). This lack of a trade-off may be related to multiple factors. First, reproductive costs are generally much lower in males than in females (Trivers, 1972) so it is difficult to detect the reproductive penalties for macropterous males (Ott, 1994). Second, short-winged (SW) males may also have a reproductive advantage over their long-winged (LW) counterparts in terms of other characteristics such as fertilization success. For example, no difference in testis mass was found between LW and SW males of Gryllus firmus Scudder (Orthoptera: Gryllidae), and there were also no differences between males of the two morphs in terms of their ability to sire offspring when pairs of males were confined permanently with single females (Roff \& Fairbairn, 1993) but flight-capable

\footnotetext{
* Corresponding author; e-mail: daohongzhu@yeah.net
} 
males sing less than flight-incapable males (Mitra et al., 2011) and have fewer mating opportunities (Crnokrak \& Roff, 1995). Compared with SW males, LW Gryllus texensis Cade \& Otte (Orthoptera: Gryllidae) have a decreased propensity for courtship (Guerra \& Pollack, 2007) and are less aggressive in agonistic encounters (Guerra \& Pollack, 2010). The SW males of Velarifictorus asperses Walker (Orthoptera: Gryllidae) have both a higher reproductive ability and fighting success than the LW males (Zeng \& Zhu, 2012; Zeng et al., 2016). These studies suggest that a trade-off between the flight capacity and reproduction does exist in wing-dimorphic male insects, but this trade-off appears to vary in different insect taxa.

The field cricket, Velarifictorus ornatus (Shirake, 1911) (Orthoptera: Gryllidae), exhibits a distinct wing dimorphism. The LW morph with hindwings extending beyond its forewings and developed flight muscles can fly whereas the SW morph with reduced wings and flight muscles cannot fly. A physiological trade-off clearly exists between flight capability and reproduction in LW and SW adult females of $V$. ornatus but their gonad sizes are similar in LW and SW males (Zhao et al., 2010). In a recent study, we found that the SW males reach sexual maturity earlier and have a higher mating frequency, greater spermatophore weight, and higher fertilization success than LW males (Zhao et al., 2016), that is, there is a trade-off between flight capability and reproduction in males of the wing-dimorphic cricket $V$. ornatus (Zhao et al., 2016). However, successfully acquiring a mating partner is essential for demonstrating that SW individuals have a reproductive advantage over their LW counterparts. Thus, in the current study, we determined the mating ability of SW and LW males when competing for mates. The aim of this study was to determine whether SW males have a mating advantage over LW males because their underdeveloped flight muscles might allow them to devote more resources to reproduction.

\section{MATERIAL AND METHODS}

\section{Insects and rearing methods}

The experimental crickets, $V$. ornatus, were obtained from an established laboratory colony, which originated from a population collected in May 2005 in Zhuzhou, China $\left(27^{\circ} 48^{\prime} \mathrm{N}, 113^{\circ} 12^{\prime} \mathrm{E}\right)$. Newly hatched nymphs were raised in groups in plastic containers $(60 \times 30 \times 20 \mathrm{~cm})$ according to a previously described protocol (Zhao et al., 2010, 2016). On the day of adult emergence, the adults were transferred into another container $(13 \times 13 \times 8.5 \mathrm{~cm})$ and kept separately under a $16 \mathrm{~L}: 8 \mathrm{D}$ photoperiod and $25^{\circ} \mathrm{C}$ prior to use in the following experiments.

\section{Experiment 1: Mating}

A previous study showed that the preoviposition period of adult females of $V$. ornatus is 9-10 days (Zhao et al., 2010) and males reach sexual maturity 6 days after adult emergence (Zhao et al., 2016). Therefore, we paired 6-day-old virgin males with 8-dayold virgin females. A SW male was first placed in a plastic container $(13 \times 13 \times 8.5 \mathrm{~cm})$ and a LW female was then placed in the same container to provide the male with a mating opportunity. The mating between the female and male was recorded using a digital camera (Nikon, D80).

\section{Experiment 2: Mating of LW and SW males when competing for females}

A 8-day-old virgin female was placed on one side of a plastic container $(60 \times 30 \times 20 \mathrm{~cm})$ and covered by a plastic cup to prevent her from moving in the container. Next, a 6-day-old virgin SW male and a 6-day-old virgin LW male were placed on the other side of the container and covered by another plastic cups, so that the SW and LW males were at an same distance from the female. At the start of the experiment, the plastic cups were removed and the female and males were allowed to move freely in the container. Mating success was defined as the transfer of a spermatophore from a male to the female during the mating. In order to clarify the difference in mating of LW and SW males, we performed four mating choice trials.

\section{Experiment 2a: Effect of wing morph of female on mating success}

A SW and a LW male were allowed to compete for a SW female and in a parallel experiment a SW and a LW male competed for a $\mathrm{LW}$ female. This was done in order to determine whether the morph of the female affected the outcome.

\section{Experiment 2b: Effect of mating experience of SW males on mating success}

A virgin LW male and a SW male that had mated with a female $2 \mathrm{~h}$ previously were allowed to compete for a female.

\section{Experiment 2c: Effect of age on mating success}

In order to investigate the effect of age on mating competitiveness, 6-, 10- and 15-day old SW males matched with the same age LW males were allowed to compete for females.

\section{Experiment 2d: Effect of de-altaion on mating success}

A LW male from which the hind-wings had been removed using forceps on the day of adult emergence and a SW male were allowed to compete for a female.

Before starting an experiment, we determined the body weight, head width, and femur length of LW and SW males, and selected similar sized LW and SW males for use in the competition experiments in order to eliminate the effects of differences in body weight on their mating competitiveness. In the four mating choice trials, we recorded the percentages of LW males and SW males that mated with females, and the duration of courtship. If the mating success of SW males was significantly higher than that of LW males, we recorded that the SW males had a mating advantage over LW males. In addition, we recorded the ratio of LW males that fought with SW males, and the probability of LW and SW males of winning in experiment $2 \mathrm{a}$. The result of experiment $2 \mathrm{a}$ showed that wing form of females had no effect on the mating advantage of SW males over LW males, so LW or SW females were used randomly in experiment $2 \mathrm{~b}, \mathrm{c}$ and $\mathrm{d}$ and experiment 3 .

\section{Experiment 3: Effect of singing on mating \\ competitiveness}

Crickets produce songs by vibrating their wings, and their ability to sing is lost if their stridulitrum is removed, but their mating behaviour is not affected (Bailey et al., 2007). To investigate the effects of singing on the mating competitiveness of males, the LW and SW males competing for a female were divided into three treatment groups: experiment $3 \mathrm{a}$, an intact LW male was paired with a SW male with the left stridulitrum removed; experiment $3 \mathrm{~b}$, an intact LW male was matched with a SW male with both its left and right stridulitra removed; experiment $3 \mathrm{c}$, the left and right stridulitra were removed from both the LW and SW males. In these three treatments, we recorded the percentages of LW males 

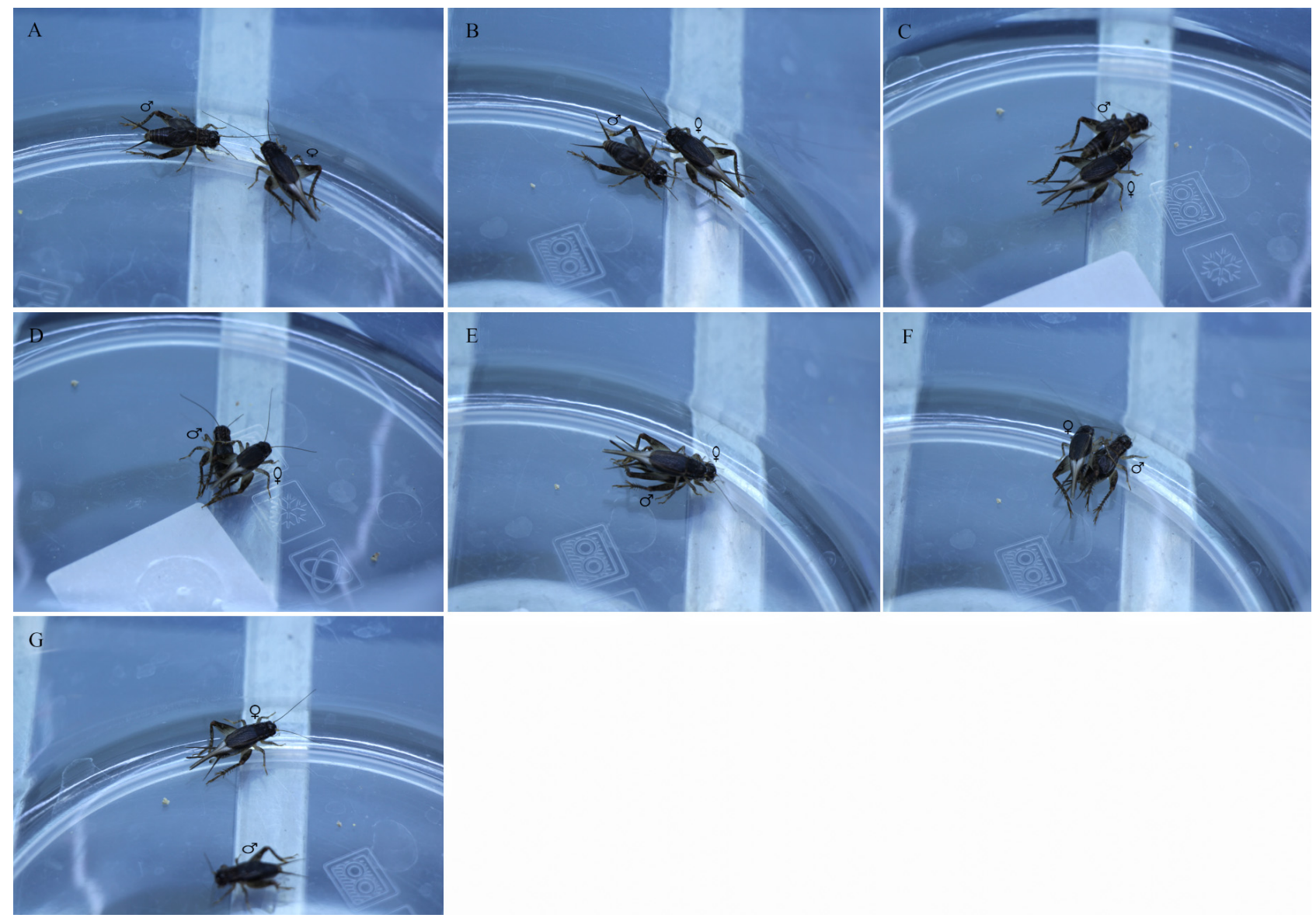

Fig. 1. Photographs of female and male Velarifictorus ornatus at different stages of mating.

and SW males that mated with females, as well as the duration of courtship.

\section{Statistical analysis}

Student's $t$-test was used to compare the body dimensions of LW and SW males. Mating success of SW and LW males in binomial choice trials was analyzed using a binomial test (the test probability was 0.5 ). The chi-squared test was used to compare mating success of virgin SW males and mated SW males, mating success of SW males competing for LW and SW females and the mating success of 6,10 and 15-day old SW males. Mann-Whitney $U$ test was used to compare courtship duration of LW and SW males in binomial choice trials. Kruskal-Wallis test was used to analyze duration of courtship among 6,10, 15 day old males and stridulitra removed males.

\section{RESULTS}

\section{Experiment 1: Mating}

After a female was transferred into the container, the male began to sing and approached the female immediately (Fig. 1A). Subsequently, the female moved close to the male in response to the singing of the male (Fig. 1B). As the female approached the male, the male moved toward the front of the female and prepared to mate with her (Fig. 1C). After the female made contact with the male, which was accompanied by the male occasionally tapping the female with his antennae, the male stopped singing and waited for the female (Fig. 1D). Next, the female climbed onto the back of the male and received the spermatophore
(Fig. 1E). The female then left the back of the male after successfully receiving the spermatophore (Fig. 1F) and then moved away from the male (Fig. 1G).

\section{Experiment 2: Mating competitiveness of LW and SW males}

\section{Experiment 2a: Effect of wing morph of female on mating success}

There were no significant differences in the body weight, head width and femur length of the LW and SW males that competed for the same females (Table 1, t-test, body weight: $t=-1.651, P=0.1$; head width: $t=-0.634$, $P=0.526$; femur length: $t=-0.138, P=0.890, \mathrm{n}=146)$. When LW and SW males competed for a female, all of them completed the mating within $15 \mathrm{~min}$ and there was no significant difference in the duration of courtship of LW and SW males (Fig. 2B, Mann-Whitney $U$ test, competing for SW females: $z=-0.908, P=0.364$; competing for LW females: $z=-1.611, P=0.107)$. However, compared with LW males, the SW males had an obvious competitive

Table 1. Comparison of the body dimensions of long-winged (LW) and short-winged (SW) adult males of Velarifictorus ornatus used in experiment $2 \mathrm{a}$.

\begin{tabular}{lccc}
\hline Wing morph Body weight $(\mathrm{mg})$ & Head width $(\mathrm{mm})$ & Femur length $(\mathrm{mm})$ \\
\hline LW & $141.79 \pm 3.58$ & $3.21 \pm 0.05$ & $7.09 \pm 0.05$ \\
SW & $138.06 \pm 3.93$ & $3.19 \pm 0.05$ & $7.02 \pm 0.08$ \\
\hline
\end{tabular}

Body dimensions between LW and SW males were compared and found to be not significantly different (t-test: $P>0.05$ ). 

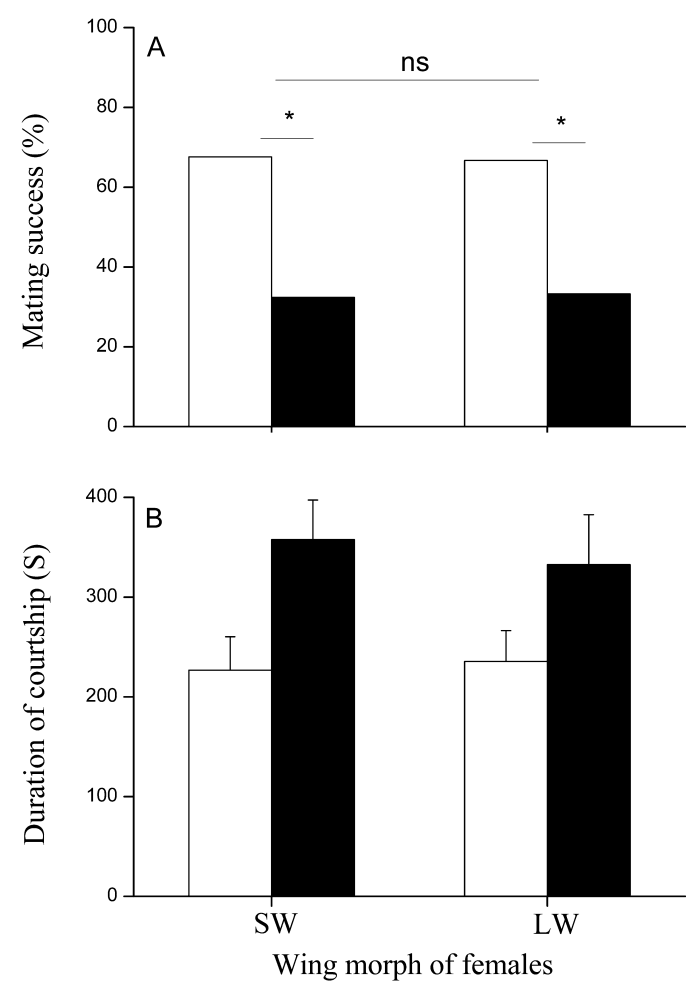

Fig. 2. Comparison of the mating success and duration of courtship (+SE) of short-winged (SW) (white bars) and long-winged (LW) (black bars) males of Velarifictorus ornatus. Asterisks indicate significant differences between SW and LW males (Binominal test, $P<0.05)$. "ns" indicates no significant difference in mating success of SW males when presented with LW and SW females separately ( $X^{2}$-test, $\left.P>0.05\right)$.

advantage, as the mating success of SW males was about $67.6 \%$, which was significantly greater than the $32.4 \%$ recorded for LW males (Fig. 2A, Binominal test, $P=0.003$, $\mathrm{n}=74$ pairs) when the males competed for a SW female. Similarly, the SW males had a significantly higher mating success than LW males (Fig. 2B, Binominal test, $P=0.006$, $\mathrm{n}=72$ pairs) when the SW females were replaced with LW females. Furthermore, there was no difference in the mating success of SW males when they competed for LW and SW females (Fig. 2A, $\chi^{2}$-test, $\chi^{2}=0.013, P=0.908$ ). In addition, the fighting success of $\mathrm{LW}$ and $\mathrm{SW}$ males was similar (Binomial test, $P=1.0$ ) when they competed for the same female. So the mating advantage of SW males over LW males recorded in this study is not determined by differences in the fighting success of LW and SW males.

\section{Experiment 2b: Effect of mating experience of SW males on mating success}

When a virgin LW male and a SW male that had mated once with a female $2 \mathrm{~h}$ previously competed for a female, the percentage mating success of the SW males $(64.2 \%)$ was significantly greater than that of LW males $(35.8 \%)$ (Fig. 3A, Binomial test, $P=0.012, \mathrm{n}=84$ pairs). In addition, the mating success of virgin SW males and SW males with mating experience was similar $\left(\chi^{2}\right.$-test, $\chi^{2}=0.032, P$ $=0.716)$. There was also no difference in the duration of courtship of LW and SW males (Fig. 3B, Mann-Whitney $U$ test, $z=-1.145, P=0.252$ ).
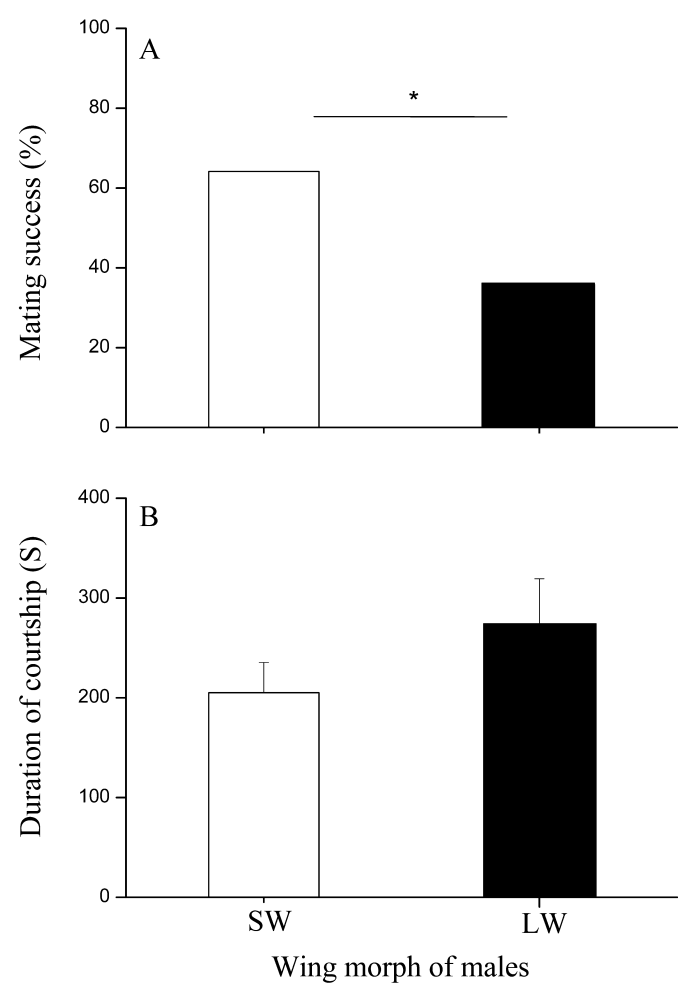

Fig. 3. Effects of mating experience of the males on the mating preference of females and duration of courtship (+SE) by longwinged (LW) and short-winged (SW) males of Velarifictorus ornatus. Asterisks indicate significant differences between SW and LW males (Binomial test, $P<0.05$ ).
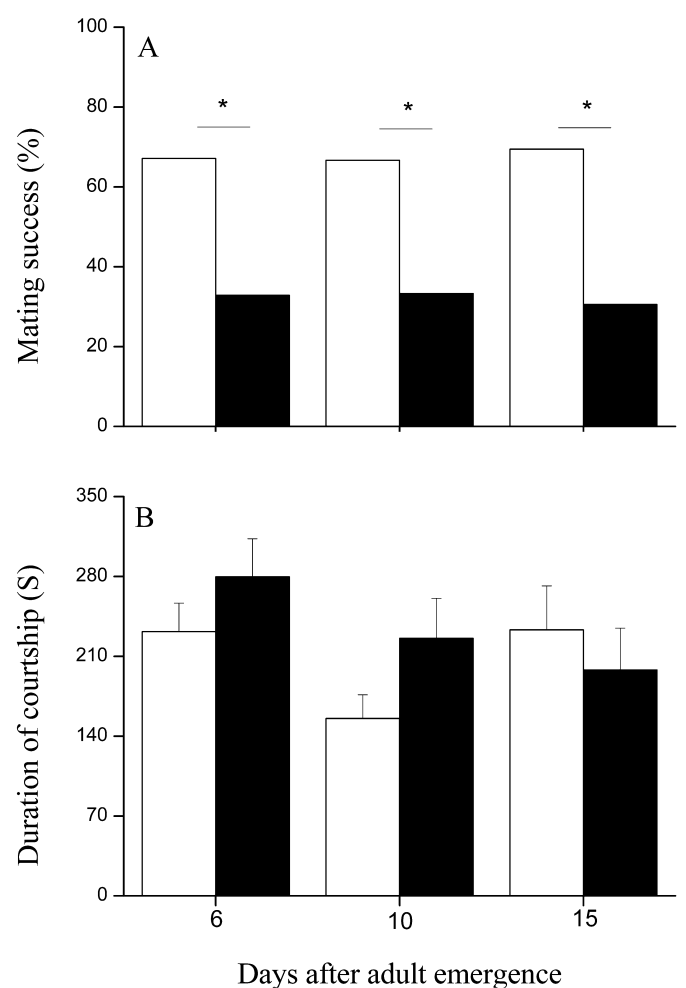

Fig. 4. Effects of male age on the mating preference of females and duration of courtship (+SE) by short-winged (SW) (white bars) and long-winged (LW) (black bars) males of Velarifictorus ornatus. Asterisks indicate significant differences between LW and SW males (Binomial test, $P<0.05$ ). 

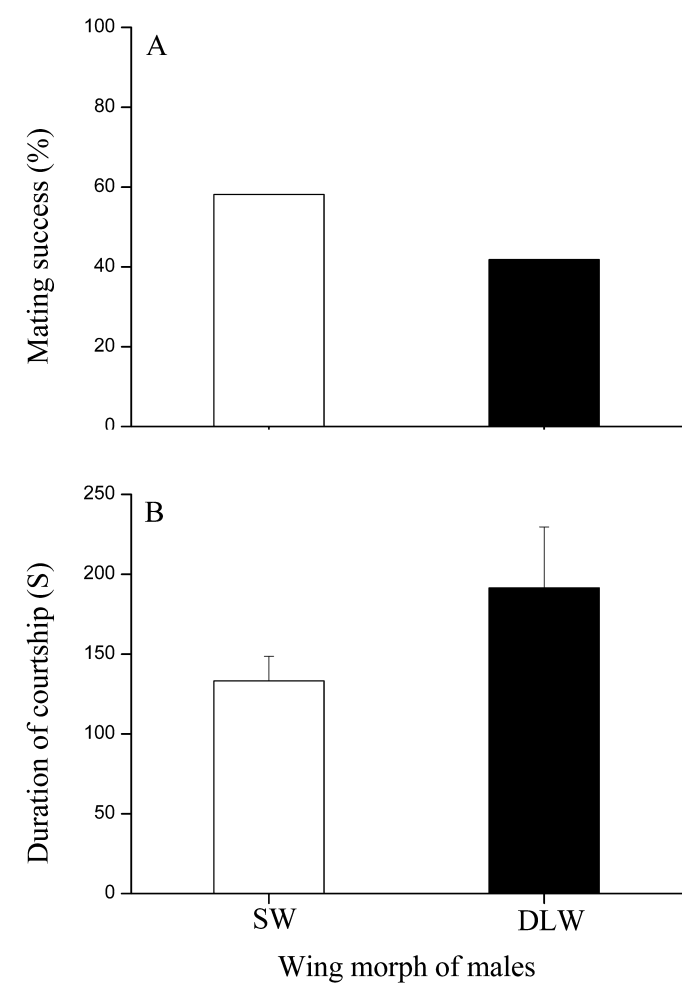

Fig. 5. Effects of dealation of long-winged (LW) males on the mating preference of females and duration of courtship (+SE) by shortwinged (SW) and dealate LW (DLW) males of Velarifictorus ornatus (Binomial test, $P>0.05$ ).

\section{Experiment 2c: Effect of age on mating success}

On day 6, 10 and 15 after adult emergence, the mating success of SW males was significantly greater than that of LW males (Fig. 4A, Binomial test, $6 \mathrm{~d}: P=0.005, \mathrm{n}=73$ pairs; $10 \mathrm{~d}: P=0.004, \mathrm{n}=78$ pairs; $15 \mathrm{~d}: P=0.001, \mathrm{n}=$ 72 pairs) and the mating success of SW males was similar in the three treatments $\left(\chi^{2}\right.$-test, $\left.\chi^{2}=0.149, P=0.92\right)$. There was also no significant difference in the duration of courtship of LW and SW males (Fig. 4B, Mann-Whitney $U$ test, $6 \mathrm{~d}: z=-1.6, P=0.11 ; 10 \mathrm{~d}: z=-1.625, P=0.104,15 \mathrm{~d}: z=$ $-0.12, P=0.904)$ and the duration of courtship of SW and LW males were also similar in the three treatments (Fig. 4B, Kruskal-Wallis test, SW males: $H_{2}=2.641, P=0.267$; LW males: $H_{2}=0.244, P=0.885$ ).

\section{Experiment 2d: Effect of de-altaion on mating success}

Dealation significantly improved the mating success of LW males as their mating success was similar to that of SW males (Fig. 5A, Binomial test, $P=0.242, \mathrm{n}=72$ pairs) and it had no effect on the duration of courtship when dealate LW males and SW males competed for the same females (Fig. 5B, Mann-Whitney $U$ test, $z=-0.973, P=0.33$ ).

\section{Experiment 3: Effect of singing on mating competitiveness}

The mating success of SW males with the left stridulitrum removed on the day of emergence was significantly greater than that of intact LW males competing for the same females (Fig. 6A, Binomial test, $P=0.038, \mathrm{n}=84$ pairs). Compared with SW males, intact LW males had a much
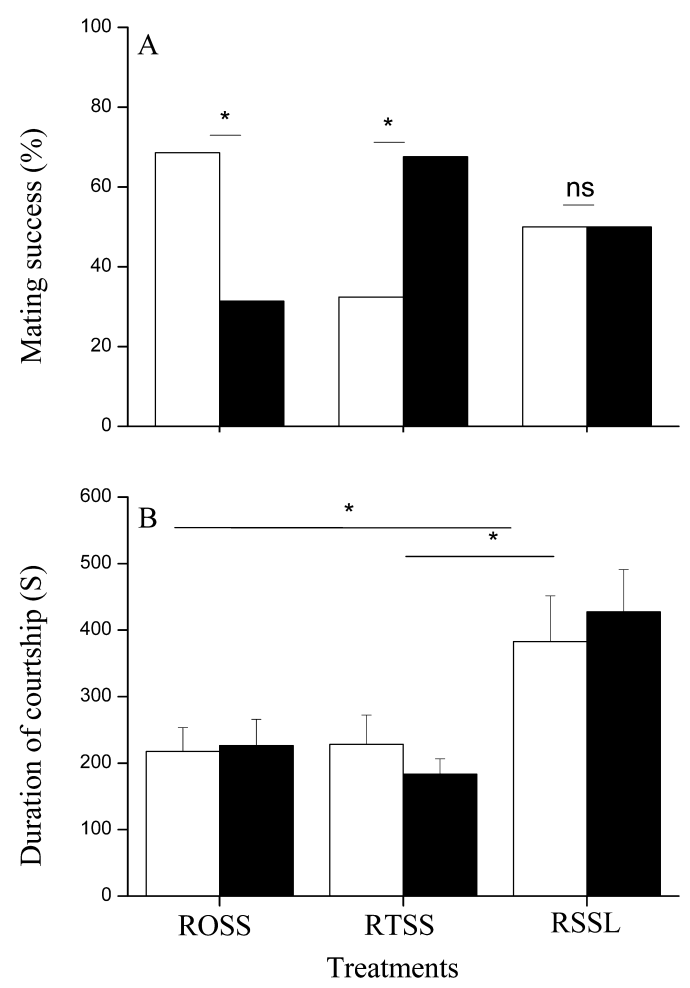

Fig. 6. Effects of stridulitra removal on the mating preference of females and duration of courtship (+SE) by short-winged (SW) (white bars) and long-winged (LW) (black bars) males of Velarifictorus ornatus. ROSS - remove one stridulitrum of SW; RTSS - remove two stridulitra of SW; RSSL - remove sridulitra both SW and LW. Asterisks indicate significant differences between SW and LW males (Binomial test for mating success; Kruskal-Wallis test for duration of courtship, $P<0.05$; ns - not significant).

greater likelihood of mating when both the left and right stridulitra of SW males were removed after adult emergence (Fig. 6A, Binomial test, $P=0.023, \mathrm{n}=86$ pairs). However, when the left and right stridulitra were removed from both SW males and LW males, the mating success of the LW and SW males was similar, and there was no significant difference between them (Fig. 6A, Binomial test, $P=0.738, \mathrm{n}=80$ pairs). The mating success and duration of courtship were both affected by the removal of the stridulitrum. There was no significant difference in duration of courtship of intact LW males and SW males with one stridulitrum or both stridulitra removed on the day of emergence (Fig. 6B, Mann-Whitney $U$ test, one stridulitrum removed: $z=-1.036, P=0.3$; two stridulitra removed: $z=$ $-1.121, P=0.262)$. However, stridulitra removal clearly increased the duration of courtship by LW and SW males compared to intact LW and SW males with one stridulitrum or two stridulitra removed (Fig. 6B, Kruskal-Wallis test, SW males: $H_{2}=7.473, P=0.026$; LW males: $H_{2}=$ $18.843, P<0.001)$, although the duration of courtship was similar in LW and SW males with stridulitra removed (Fig. 6B, Mann-Whitney $U$ test, $z=-1.611, P=0.107$ ).

\section{DISCUSSION}

The successful acquisition of a mate is necessary for males to produce offspring. Mating in V. ornatus is as follows, a male encounters a female, the male sings to attract 
the female, which if she chooses to mate with the male then climbs onto the back of the male and accepts a spermatophore (Fig. 1), at which stage they have mated successfully. Some females after approaching males, however, turn away and do not mate with them (data not shown), thereby indicating that mate selection occurs before a female decides to mate. For successful mating, females need to voluntarily climb on the back of a male, and thus the success or failure of mating is determined by the female choosing her mate rather than a female simply encountering a male.

Males often produce sex signals made up of many components, any of which might decide the success or failure of mating by males (Stoffer \& Walker, 2012). In the mating process in $V$. ornatus, the male attracts a female by singing after they encounter one another (Fig. 1), but SW males were preferentially chosen by females (Fig. 2). This result is similar to that reported for Pyrrhocoris apterus L. (Heteroptera: Pyrrhocoridae), in which the mating success of SW males is also significantly greater than that of LW males (Socha, 2004). However, unlike in Prokelisia dolus Wilson (Hemiptera: Delphacidae), where the competitive mating advantage of SW males is related to the wing morph of the females (Langellotto el al., 2000), both LW and SW $V$. ornatus females preferred SW males, so the mating advantage of SW males over LW males was not affected by the wing form of the females (Fig. 2). Females mating with virgin males may obtain larger spermatophores and more sperm to fertilize their eggs resulting in greater fecundity (Jiménez-Pérez \& Wang, 2004; King $\&$ Fischer, 2009) and therefore females preferentially mate with virgin males (Monceau \& van Baaren, 2012; Muller et al., 2016). The weight of spermatophore obtained by mating with experienced males is lighter than that obtained from virgin males (Zhao et al., 2016). The mating ability of SW males, however, was not affected by mating experience (Fig. 3). In addition, age has no effect on the mating ability of SW males (Fig. 4). Thus, we infer that the enhanced mating advantage of SW males, compared to that of their LW counterparts, is associated with the loss of the ability to fly, whereas LW males retain the ability to fly at the expense of a reduced mating capacity.

A trade-off between flight-muscle development and reproductive success exists in wing-dimorphic males of $V$. ornatus (Zhao et al., 2016). In addition, we found that the mating ability of SW males was significantly greater than that of LW males (Fig. 2). The enhanced mating ability of SW males may also be related to differences in the allocation of resources to flight muscles and reproductive organs in LW and SW males. After adult emergence, the weight of the flight muscles of LW males increases significantly and reaches a maximum on day 3 and then decreases on day 5 . In contrast, the flight muscles of SW males remain constant and are significant lighter than those of LW males (Zhao et al., 2010). Both the development of flight muscles and the production of sound require energy (Wagner, 2005; Mowles, 2014), but in LW males, the flight muscles consume much more energy than those of SW males. That is, in not developing flight muscles SW males can allocate more resources to the development of their reproductive organs and producing a more attractive sex signal in terms of their song, which gives SW males a mating advantage. The above speculation was validated by the enhanced mating ability of dealated LW males (Fig. 5). After removing the hind-wings, the flight muscles of LW individuals degenerate and their weight is similar to those in SW individuals (Tanaka, 1994; Zhao et al., 2010). The dealated LW males do not need to develop and maintain their flight muscles (Tanaka, 1991), so can allocate more resources to developing their reproductive organs and producing a more attractive song. As a result, the mating success of dealate males was similar to that of SW males when competing for the same females (Fig. 5). These findings are consistent with those recorded for the cricket G. firmus, in which dealation also increases the mating success of LW males (Mitra et al., 2011).

The mating success of males can also be affected by their nutritional status and age (Weddle et al., 2012; Wang et al., 2016). At 6, 10 and 15 days after adult emergence, the mating abilities of SW males were significantly greater than that of LW males, and thus the mating advantage of SW males over LW males was not affected by age (Fig. 4). On day 3 after adult emergence, the flight muscles reach their maximum development in LW males, and then begin to degrade but the degraded flight muscles in LW males are still significantly heavier than those in SW males (Zhao et al., 2010). The development of flight muscles and their maintenance consumes large amounts of energy (Tanaka, 1993). Compared with SW males, LW males must allocate more resources than SW males to flight muscle maintenance, whereas SW males can allocate more resources to the production of attractive songs. Thus, the mating ability of SW males will be greater than that of LW males 10 and 15 days after adult emergence.

In many organisms, songs play a role in reproductive isolation, sexual selection, and territoriality (Doherty, 1985). For example, flightless morphs of G. firmus sing more than the flight-capable morph and -flight muscle histolysis increases the mating success of long-winged males because it increases singing activity (Mitra et al., 2011). In this study, when only one stridulitrum was removed from SW males and they were still able to produce songs, the SW males still had an obvious mating advantage over LW males (Fig. 6). When both stridulitra were removed from SW males, however, they lost the ability to produce songs and their mating advantage over LW males (Fig. 6). These results indicate that the mating advantage of SW males over LW males depends on the greater attractiveness of the songs produced by SW males. This hypothesis was validated by removing both stridulitra from LW and SW males. When both stridulitra were removed from LW and SW males, thereby eliminating their ability to produce songs, the LW and SW males had similar mating successes (Fig. 6). In addition, the duration of courtship was significantly prolonged after stridulitra removal (Fig. 6). The females of the sagebrush cricket, Cyphoderris strepitans Morris \& Gwynne (Orthoptera: Haglidae) only mate with males that 
produce songs (Snedden \& Sakaluk, 1992). Based on the results of the present study, the difference in mating success of LW and SW males can be eliminated and duration of courtship extended, but nevertheless all the males successfully mated with females. These results indicate that the successful mating by females of $V$. ornatus depends on the sex signals males produce not only in terms of singing but also other signals. In field crickets, the males produce various signals that might affect mating decisions, including different types of songs, tactile signals and chemical signals (Tregenza \& Wedell, 1997). However, the SW males of $V$. ornatus have a much stronger competitive ability than LW males due to their songs and females choose their mates based on the differences in the songs of LW and SW males.

The mating ability of males can be affected by their body weight (Deb et al., 2012), age (Verburgt et al., 2010) and other factors. In the present study, we paired LW and SW males of similar ages and body weights in the same treatment (Table 1), but the females nevertheless preferred SW males as mating partners (Fig. 2). Thus, the mating advantage of SW male over LW males was not affected by the size and age of the individuals. Females may prefer to mate with SW males because of reproductive benefits. Compared with LW males, SW males produce heavier spermatophores and the success of fertilization by SW males is significantly greater than that by LW males (Zhao et al., 2016). Thus, females that preferentially mate with SW males can improve their likelihood of successful fertilization and produce more offspring and thereby increase their fitness.

ACKNOWLEDGEMENT. This work was supported by National Nature Science Foundation of China (no. 31200494).

\section{REFERENCES}

Bailey N.W., Gwynne D.T., Bailey W.V. \& Ritche M.G. 2007: Multiple differences in calling songs and other traits between solitary and gregarious Mormon crickets from allopatric mtDNA clades. - BMC Evol. Biol. 7: 5, 11 pp.

CRNOKRAK P. \& RofF D.A. 1995: Fitness differences associated with calling behavior in the two morphs of male sand cricket, Gryllus firmus. - Anim. Behav. 50: 1475-1481.

Deb R., Bhattacharya M. \& Balakrishnan R. 2012: Females of a tree cricket prefer larger males but not the lower frequency male calls that indicate large body size. - Anim. Behav. 84: 137-149.

DOHERTY J.A. 1985: Trade-off phenomena in calling song recognition and phonotaxis in the cricket, Gryllus bimaculatus (Orthoptera, Gryllidae). - J. Compar. Physiol. (A) 156: 787-801.

GuERRA P.A. 2011: Evaluation the life-history trade-off between dispersal capability and reproduction in wing dimorphic insects: A meta-analysis. — Biol. Rev. 86: 813-835.

Guerra P.A. \& Pollack G.S. 2007: A life history trade-off between flight ability and reproductive behavior in male field crickets (Gryllus texensis). - J. Insect Behav. 20: 377-387.

Guerra P.A. \& Pollack G.S. 2010: Colonists and desperadoes: Different fighting strategies in wing-dimorphic male Texas field crickets. - Anim. Behav. 79: 1087-1093.

HARRISON R.G. 1980: Dispersal polymorphisms in insects. Annu. Rev. Ecol. Syst. 11: 95-118.
JimÉNEZ-PÉREZ A. \& WANg Q. 2004: Sexual selection in Cnephasia jactatana (Lepidoptera: Tortricidae) in relation to age, virginity, and body size. - Ann. Entomol. Soc. Am. 97: 819-824.

KING B.H. \& FISCHER C.R. 2009: Male mating history: effects on female sexual responsiveness and reproductive success in the parasitoid wasp Spalangia endius. - Behav. Ecol. Sociobiol. 64: 607-615.

Langellotto G.A., Denno R.F. \& Ott J.R. 2000: A trade-off between flight capability and reproduction in males of a wingdimorphic insect. - Ecology 81: 865-875.

Mishiro K., Fujisaki K. \& NaKasuji F. 1994: Comparison of female reproductive effort and male mating success between macropterous and brachypterous forms of the small brown planthopper, Laodelphax striatellus (Homoptera: Delphacidae). - Appl. Entomol. Zool. 29: 211-217.

Mitra C., Wagner W.E., Zera A.J. \& Tolle A.E. 2011: Variation in singing behaviour among morphs of the sand field cricket, Gryllus firmus. - Ecol. Entomol. 36: 152-160.

Monceau K. \& van BAaren J. 2012: Female teneral mating in a monandrous species. - Ecol. Evol. 2: 1426-1436.

Mowles S.L. 2014: The physiological cost of courtship: field cricket song results in anaerobic metabolism. - Anim. Behav. 89: $39-43$.

Muller K., Arenas L., Thiéry D. \& Moreau J. 2016: Direct benefits from choosing a virgin male in the European grapevine moth, Lobesia botrana. - Anim. Behav. 114: 165-172.

Отт J.R.1994: An ecological framework for the study of planthopper mating systems. In Denno R.F. \& Perfect T.J. (eds): Planthoppers: Their Ecology and Management. Chapman and Hall, New York, pp. 234-254.

RoFf D.A. 1986: The evolution of wing dimorphism in insects. Evolution 40: 1009-1020.

RofF D.A. \& FaIrbairn D.J. 1991: Wing dimorphisms and the evolution of migratory polymorphisms among the insects. Am. Zool. 31: 243-251.

RofF D.A. \& FAIRBAIRN D.J. 1993: The evolution of alternate morphologies: Fitness and wing morphology in male sand crickets. - Evolution 47: 1572-1584.

SACK C. \& Stern D. 2007: Sex and death in the male pea aphid, Acyrthosiphon pisum: the life-history effects of a wing dimorphism. - J. Insect Sci. 7: 45, 9 pp.

SNEDDEN W.A. \& SAKALUK S.K. 1992: Acoustic signalling and its relation to male mating success in sagebrush crickets. - Anim. Behav. 44: 633-639.

SochA R. 2004: Decreased mating propensity of macropterous morph in a flightless wing-polymorphic insect, Pyrrhocoris apterus (Heteroptera). — Eur. J. Entomol. 101: 539-545.

Stoffer B. \& Walker S.E. 2012: The use of multimodal communication in mate choice decisions by female house crickets, Acheta domesticus. - Anim. Behav. 83: 1131-1138.

TANAKA S. 1991: De-alation and its influences on egg production and flight muscle histolysis in a cricket (Velarifictorus parvus) that undergoes inter-reproductive migration. - J. Insect Physiol. 37: 517-523.

TANAKA S. 1993: Allocation of resources to egg production and flight muscle development in a wing dimorphic cricket, Modicogryllus confirmatus. - J. Insect Physiol. 39: 493-498.

TANAKA S. 1994: Evolution and physiological consequences of de-alation in crickets. - Res. Popul. Ecol. 36: 137-143.

Tregenza T. \& Wedell N. 1997: Definitive evidence for cuticular pheromones in a cricket. - Anim. Behav. 54: 979-984.

Trivers R.L. 1972: Parental investment and sexual selertion. In Campbell B. (ed.): Sexual Selection and the Descent of Man. Aldine, Chicago, IL, pp. 52-95. 
WAGNER W.E. JR. 2005: Male field crickets that provide reproductive benefits to females incur higher costs. - Ecol. Entomol. 30: $350-357$.

Verburgt L., Ferreira M. \& Ferguson J.W.H. 2011: Male field cricket song reflects age, allowing females to prefer young males. - Anim. Behav. 81: 19-29.

Wang D., Wang C.L., Singh N., Cooper R., Zha C. \& Eiden A.L. 2016: Effect of mating status and age on the male mate choice and mating competency in the common bed bug, Cimex lectularius (Hemiptera: Cimicidae). - J. Econ. Entomol. 109: $1333-1340$

Weddle C.B., Mitchell C., Bay S.K., Sakaluk S.K. \& Hunt J. 2012: Sex-specific genotype-by-environment interactions for cuticular hydrocarbon expression in decorated crickets, Gryllodes sigillatus: implications for the evolution of signal reliability. - J. Evol. Biol. 25: 2112-2125.
Zera A.J. \& Denno R.F. 1997: Physiology and ecology of dispersal polymorphism in insects. - Annu. Rev. Entomol. 42: 207-231.

ZeNG Y. \& ZHU D.H. 2012: Trade-off between flight capability and reproduction in male Velarifictorus asperses crickets. Ecol. Entomol. 37: 244-251.

ZENG Y., Zhu D.H. \& KANG W.N. 2016: Variation in fighting strategies in male wing-dimorphic crickets (Gryllidae). - Behav. Ecol. Sociobiol. 70: 429-435.

Zhaо L.Q., Zhu D.H. \& ZeNG Y. 2010: Physiological trade-offs between flight muscle and reproductive development in the wing-dimorphic cricket Velarifictorus ornatus. - Entomol. Exp. Appl. 135: 288-294.

Zhaо L.Q., Chai H.L. \& Zhu D.H. 2016: Potential reproductive advantage of short-over long-winged adult males of the cricket Velarifictorus ornatus. — Evol. Biol. 44: 91-99.

Received February 14, 2017; revised and accepted April 27, 2017 Published online May 29, 2017 